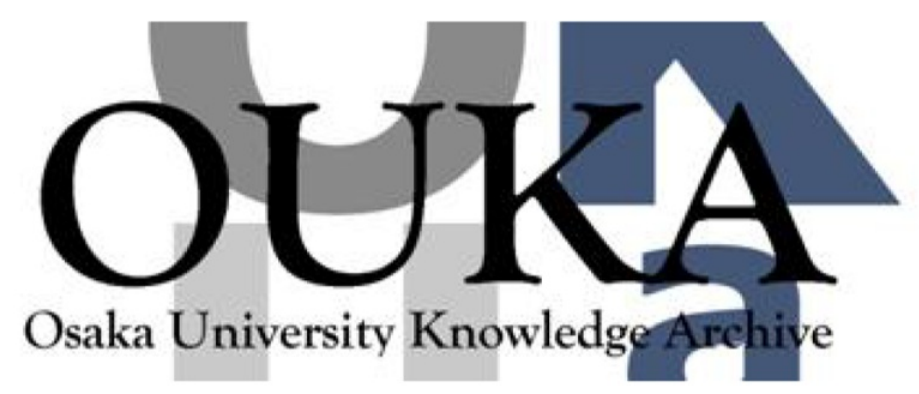

\begin{tabular}{|c|l|}
\hline Title & $\begin{array}{l}\text { Optical implementation of visible gray-image } \\
\text { morphology with the visual-area-coding } \\
\text { technique }\end{array}$ \\
\hline Author(s) & $\begin{array}{l}\text { Konishi, Tsuyoshi; Taniguchi, Shuji; Tanida, } \\
\text { Jun et al. }\end{array}$ \\
\hline Citation & Applied Optics. 35(8) p. 1234-p. 1239 \\
\hline Issue Date & $1996-03-10$ \\
\hline oaire:version & VoR \\
\hline URL & https://hdl. handle.net/11094/3441 \\
\hline rights & \\
\hline Note & \\
\hline
\end{tabular}

Osaka University Knowledge Archive : OUKA

https://ir. Library. osaka-u. ac. jp/

Osaka University 


\title{
Optical implementation of visible gray-image morphology with the visual-area-coding technique
}

\author{
Tsuyoshi Konishi, Shuji Taniguchi, Jun Tanida, and Yoshiki Ichioka
}

\begin{abstract}
We present a novel scheme of visible gray-image morphology with the visual-area-coding technique (VACT). The VACT is a technique of digitized analog-optical computing in which data are converted into visible coded patterns and processed with the visible form. Because the achievable operations in the VACT are identical to those of mathematical morphology, mathematical morphology is adapted to gray-image morphology with the VACT. Computer simulation and optical experiments of the several operations in mathematical morphology verify the correctness of the proposed technique. The processing capacity of the proposed method is estimated in terms of the space-bandwidth product.

Key words: Visualization, digitized analog-optical computing, digital halftoning, gray-image morphology, mathematical morphology. (๑) 1996 Optical Society of America
\end{abstract}

\section{Introduction}

Several techniques for implementing optical parallel logic are proposed that use the parallelism of light. ${ }^{1-4}$ Although parallelism and high-speed propagation are often emphasized as the main features of light, light has other attractive features, such as visibility for a human being. Using this feature, we propose the visual-area-coding technique (VACT) based on an area-coding technique and digital halftoning. ${ }^{5}$ We also present its application to fuzzy logic. The VACT is expected to be utilized as a technique in a human-aid system because of its visualization capability.

Mathematical morphology is an important technique for nonlinear image processing that has been used in various kinds of image processing and computer vision. ${ }^{6}$ Because the fundamental operations of mathematical morphology are achieved by convolution and threshold, several optoelectronic implementations have been proposed that make good use of the excellent features of both optics and electronics. $^{7-15}$ Because of the simplicity of its optical setup, an optoelectronic system is expected to have advan-

The authors are with the Department of Applied Physics, Faculty of Engineering, Osaka University, 2-1 Yamadaoka, Suita, Osaka 565, Japan.

Received 19 June 1995; revised manuscript received 25 September 1995.

0003-6935/96/081234-06 $\$ 06.00 / 0$

(C) 1996 Optical Society of America tages in performance and cost over electronic implementations of morphological processing.

As a matter of fact, the fundamental operations of the VACT are identical to those of mathematical morphology. This means that mathematical morphology can be used for image morphology based on the VACT. From the point of view of human interface, the VACT provides an excellent feature for visualizing the processing result for a human being. Thus we can develop a novel scheme of visible image morphology by the technique.

To clarify the capability of the VACT in image morphology, we study the application of the VACT to gray-image morphology with mathematical morphology. In this paper, we show the capability of the VACT in gray-image morphology. Section 2 explains the principles of the VACT and mathematical morphology. Section 3 describes computer simulation and optical experiments of visible gray-image morphology with the VACT. In Section 4, we discuss the processing capacity of the proposed grayimage morphology in terms of the space-bandwidth product (SBWP).

\section{Visual-Area-Coding Technique and Mathematical Morphology}

The VACT is a technique of digitized analog optical computing in which data are converted into visible coded patterns and processed with the visible form. The VACT is based on the area-coding technique ${ }^{15}$ and the microfont method. ${ }^{16}$ The area-coding technique was originally proposed as a technique for 


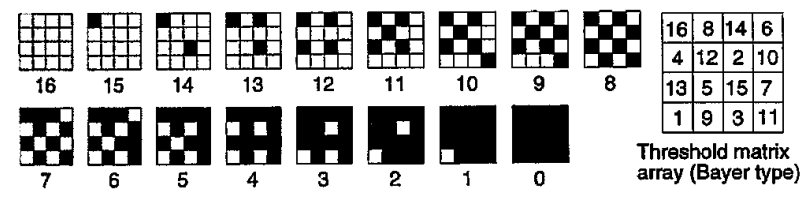

Fig. 1. Visual area codes for 17 levels.

optical parallel implementation of fuzzy logic, whereas the microfont method is a technique used in digital halftoning. Because both techniques represent a gray value as a transparent area within a pixel region, the microfont method is expected to be useful as a coding method for general-purpose computing. From this consideration, we have proposed the VACT.

In the VACT, a number is represented by a set of patterns, as shown in Fig. 1. Although Fig. 1 shows the case of 17 levels, the set for different numbers of levels can be defined according to the Bayer-type font set in the microfont method. ${ }^{17}$ Figure 2 shows the processing procedure of the maximum, minimum, and negation operations. The processing procedure consists of encoding, inversion, correlation, and rearrangement.

To illustrate the procedure in the VACT, we explain the minimum operation for two horizontally adjacent pixels. First, an input gray image is converted into a coded one. An individual pixel datum is compared with an array of multiple reference signals arranged in a threshold matrix array, whose arrangement determines the set of coded patterns shown in Fig. 1. Then the coded image is inverted onto a logic value. The inverted image is correlated with a configuration kernel to overlap the patterns of the adjacent pixels. After correlation, a logical inversion is executed to get the final result of the minimum operation again. To specify the operation for more pixels at arbitrary positions, we use arbitrary configuration kernels that correspond to the location of the pixels in the neighborhood area. For maximum operation, logical inversion is omitted, and for negation, rearrangement is required.

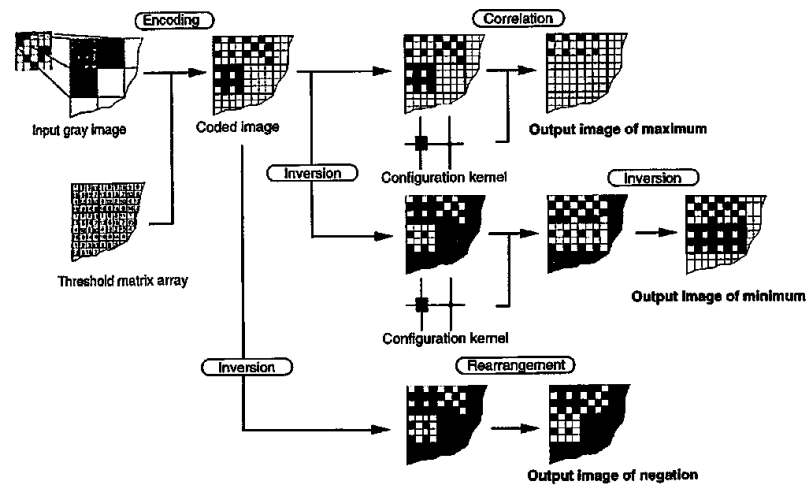

Fig. 2. Processing procedure for optical parallel implementation of maximum, minimum, and negation operations with the VACT. The shaded area in the configuration kernel indicates the center of the neighborhood area.
Mathematical morphology is an effective technique for both binary and gray-image processing, including noise reduction, edge detection, skeltonization, and size and shape extraction. The fundamental operations of mathematical morphology are dilation and erosion. Figure 3 illustrates the functions of erosion and dilation for a binary image $\mathrm{X}$ with a binary structuring element B. For binary image processing, these operations correspond to maximum and minimum operations between pixels in the neighborhood, which can be achieved by convolution between a binary image and a binary structuring element and threshold operation. For gray-image processing, morphological operations are applied after a gray image is converted into a binary one by threshold decomposition. ${ }^{18}$

Figure 4 shows a comparison of the procedure of dilation in mathematical morphology and a maximum operation in the VACT. We illustrate the

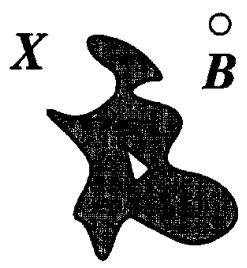

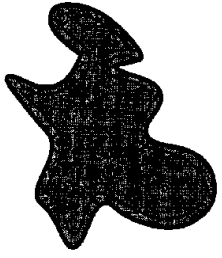

Dilation

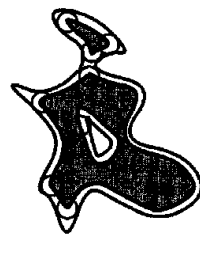

Erosion
Fig. 3. Fundamental operations of mathematical morphology: erosion and dilation of a binary image $\mathrm{X}$ by a binary structuring element B.

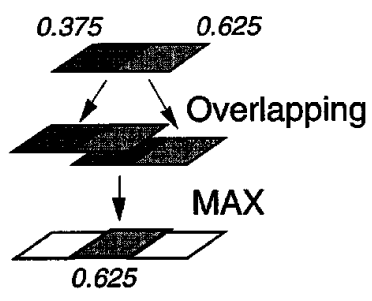

(a)

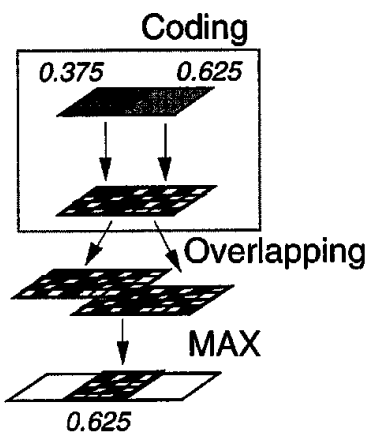

(b)
Fig. 4. Comparison of the dilation procedure in mathematical morphology and the maximum operation in the VACT: (a) dilation in mathematical morphology, (b) maximum operation in the VACT.

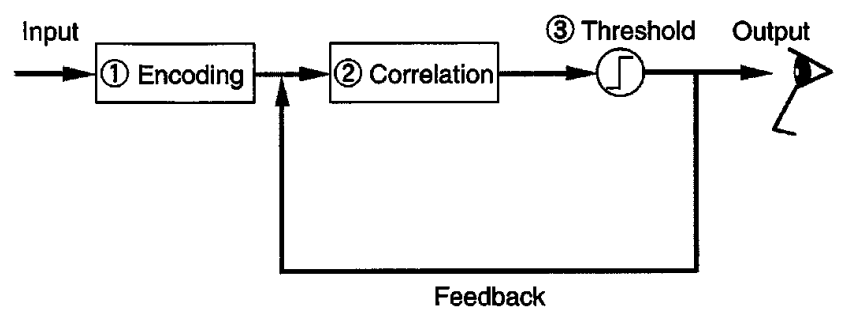

Fig. 5. Sequence of gray-image processing with the VACT. 
operation for two horizontally adjacent pixels whose values are 0.625 and 0.375 , respectively. In the VACT, each pixel is coded to a binary pattern. Because this coding process corresponds to a halftoning process, the coded pattern is able to visualize the gray value for a human visual system. As shown in Fig. 4, the fundamental operations of the VACT are basically the same as the mathematical morphology, except that results of operations in the VACT are obtained as visible coded patterns.

\section{Gray-Image Processing Based on Mathematical Morphology with the Visual-Area-Coding Technique}

Because the fundamental operations of the VACT can be used for mathematical morphology, we consider application of the VACT to gray-image morphology with mathematical morphology. In the course of the study, we put great emphasis on the visibility of the VACT. With this attractive feature, data in gray-image morphology are directly observed by human visual system.

Figure 5 shows a sequence of the gray-image morphology by the VACT. First, a target-discrete

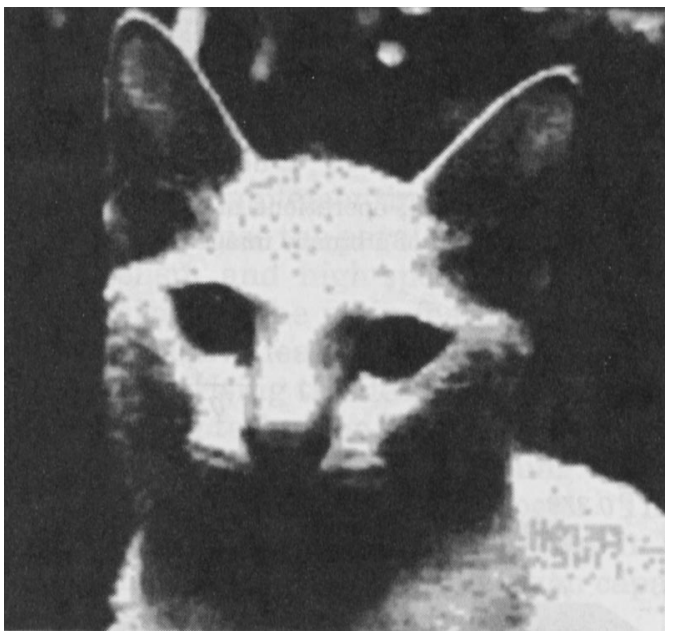

(a)

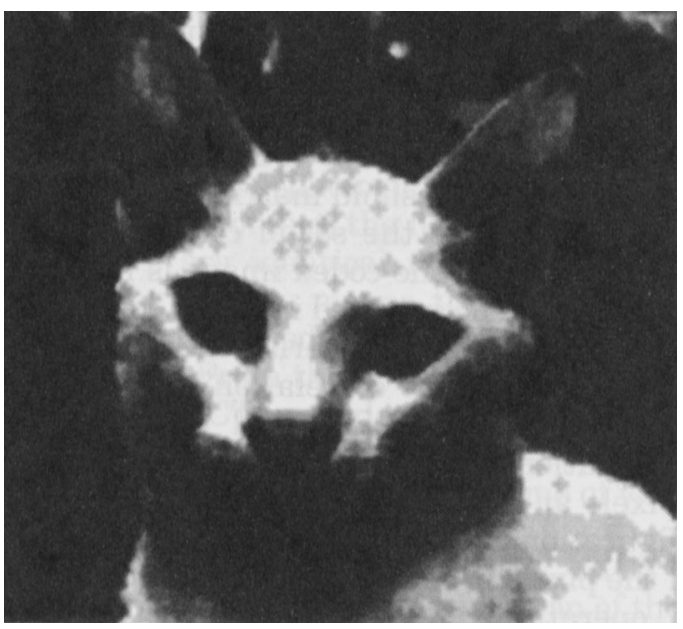

(b) continuous-tone image is transformed into a coded one. Then a discrete correlation is executed between the coded image and a configuration kernel. The dot pattern on the configuration kernel corresponds to the binary structuring element in mathematical morphology. For erosion, the contrast of the coded image is inverted before discrete correlation. The result of dilation is directly obtained after thresholding, whereas additional contrast inversion is required for erosion. All data in this sequence can be recognized by the human visual system. In addition, the result can be used as the input of the next operation without encoding, which means cascadability of the processing. These whole processes can be implemented optically as follows. Coding can be implemented by the use of a contact screen that corresponds to a threshold matrix array. Discrete correlation can be implemented by an optical correlator. Contrast inversion can be implemented by a cross Nicol arrangement of a spatial light modulator and a polarizing beam splitter. Thresholding can be implemented with a spatial light modulator.

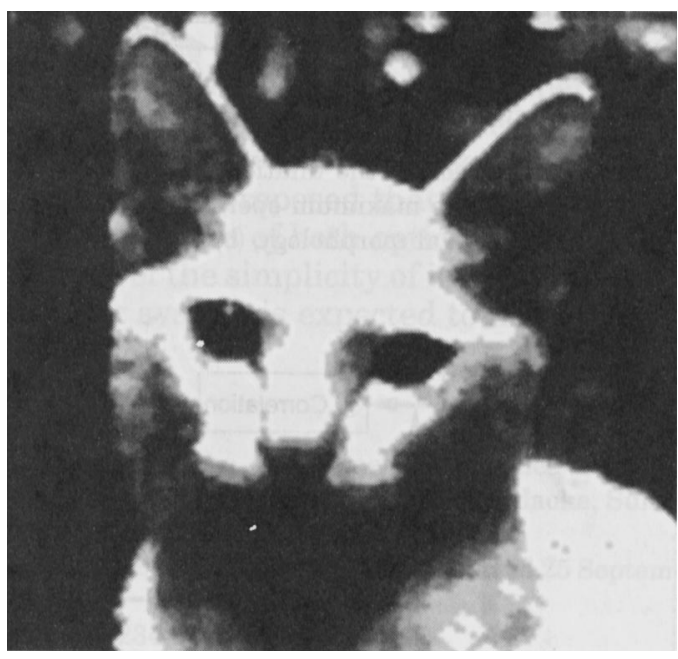

(c)

Fig. 6. Simulations of fundamental operations for gray-image processing with the VACT: (a) input image, (b) dilation, (c) erosion. The binary discrete structuring element is a rhombus. 
To verify the principle of the gray-image morphology by the VACT, we executed a computer simulation of fundamental operations and several kinds of image morphology. We used the Bayer-type font set for 17 levels as the code set of the VACT. The target image consists of $128 \times 128$ pixels with 17 levels, which is converted into a coded one with $512 \times 512$ binary pixels. As the binary discrete structuring element, a rhombus type was used. ${ }^{6}$ Figures 6(a), $6(\mathrm{~b})$, and 6(c) show the input image and the simulated results of erosion and dilation, respectively. Figure 7 shows a simulation of noise reduction. Figure 7(a) is an input image in which black and white noises are induced. Figure $7(\mathrm{~b})$ is the result of noise reduction. Figures $8(a)$ and $8(b)$ show the input image and the simulated result of edge detection, respectively. Note that we can clearly recognize the results of gray-image morphology without any decoding processes from Figs. 6-8.

As an optical implementation of the gray-image

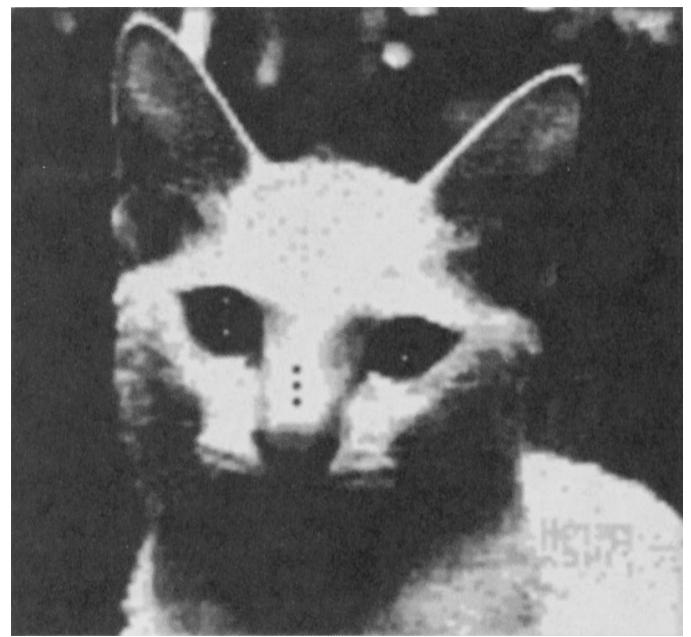

(a)

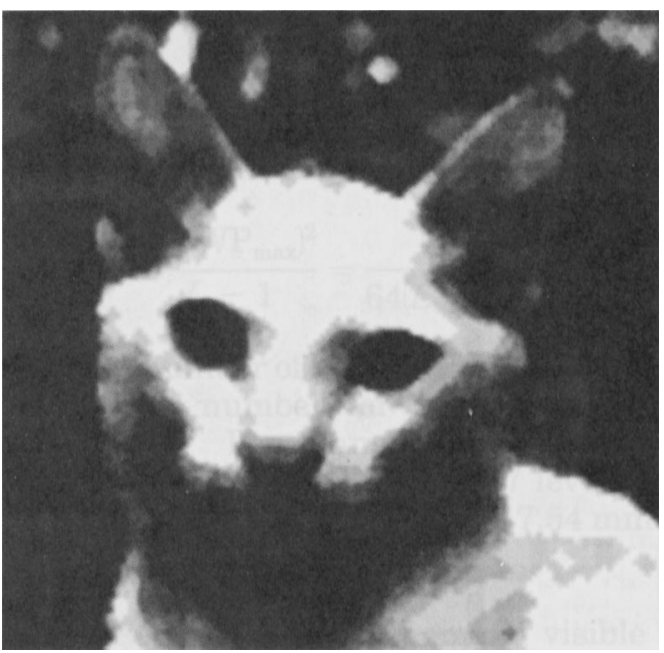

(b)

Fig. 7. Simulations of noise reduction with the VACT: (a) input image for noise reduction, (b) simulated result of noise reduction. The binary discrete structuring element is a rhombus.

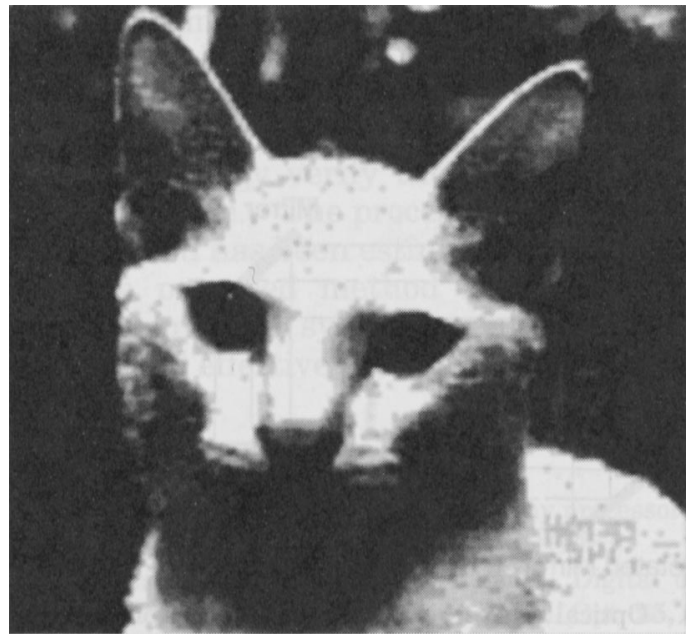

(a)



(b)

Fig. 8. Simulations of edge detection with the VACT: (a) input image for edge detection, (b) simulated result of edge detection. The binary discrete structuring element is a rhombus.

morphology by the VACT, we executed optical experiments of erosion and dilation. Figure 9 shows the experimental setup that consists of a 4 -f spatialfiltering system with a multiplex holographic filter. The impulse response of the holographic filter corresponds to the structuring element in mathematical morphology. On the holographic filter, a set of Fourier transforms of point sources, i.e., a set of

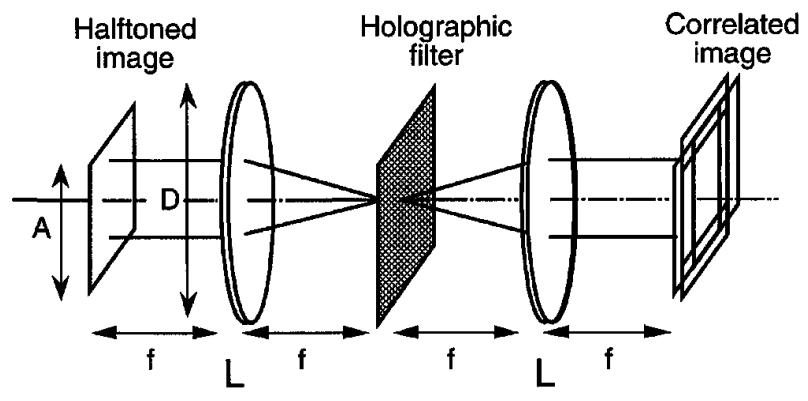

Fig. 9. Experimental system for correlation. 


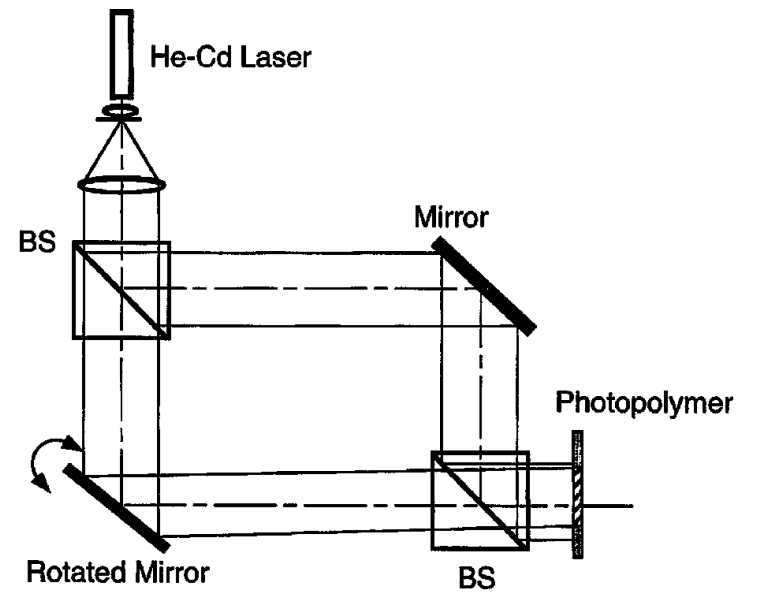

Fig. 10. Optical setup for recording the holographic filter. BS's, beam splitters.

gratings, is recorded. We used a Mach-Zehnder type of interferometer for recording the holographic filter shown in Fig. 10. The He-Cd laser $(\lambda=441.6$ $\mathrm{nm}$ ) was used to record the filter on photopolymer

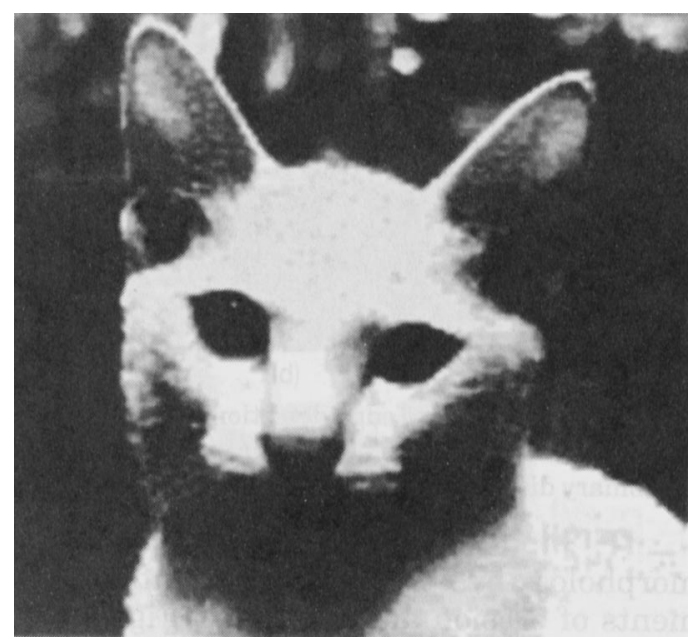

(a)

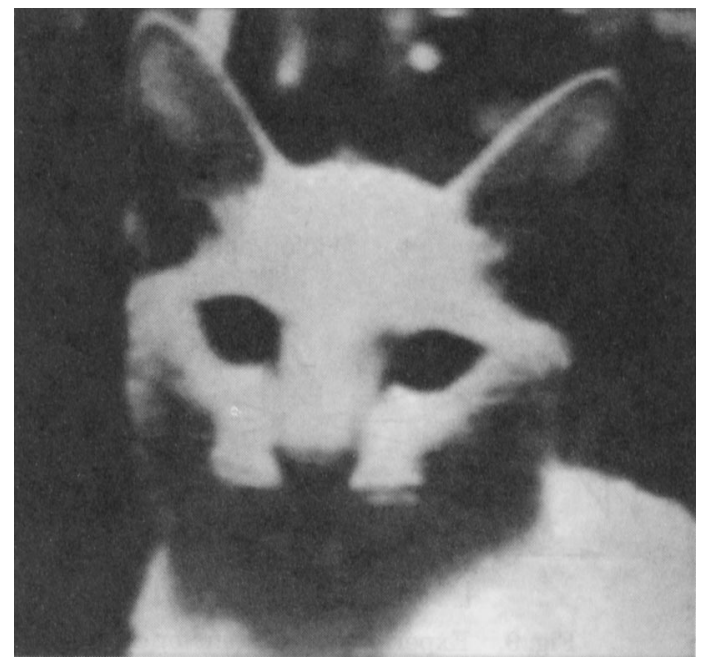

(b) material. ${ }^{19}$ The rotated mirror was prepared to configure the structuring element on the holographic filter. We generated the coded image with a computer and recorded it on a photographic film. Figures $11(\mathrm{a}), 11(\mathrm{~b})$, and $11(\mathrm{c})$ show the input image and the experimental results of erosion and dilation, respectively. By comparing Figs. 6 with Figs. 11, we can verify the correctness of the proposed technique.

\section{Discussion}

The most salient feature of the VACT is the visibility of processing data. With this feature, no special process or hardware is required for displaying the result to the human visual system. This fact results in a reduction of processing time and fabrication costs. The visible feature of the VACT can be applied to a human aid such as one that helps a doctor to inspect a large amount of medical images. In addition, we can monitor the status of processing data at any time during processing because all data in the system are visible. This feature provides novel functions for computing systems, such as the

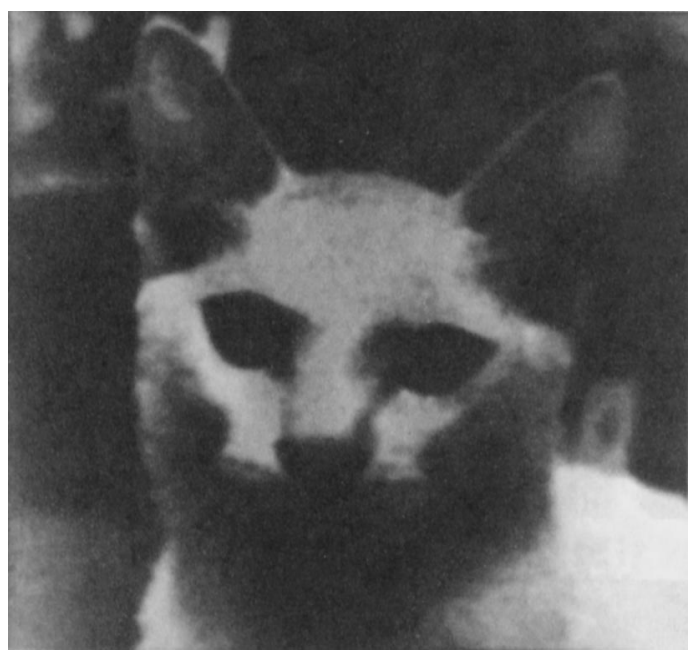

(c)

Fig. 11. Experimental results of two fundamental operations for gray-image processing with the VACT: (a) input image, (b) dilation, (c) erosion. The binary discrete structuring element is a rhombus. 


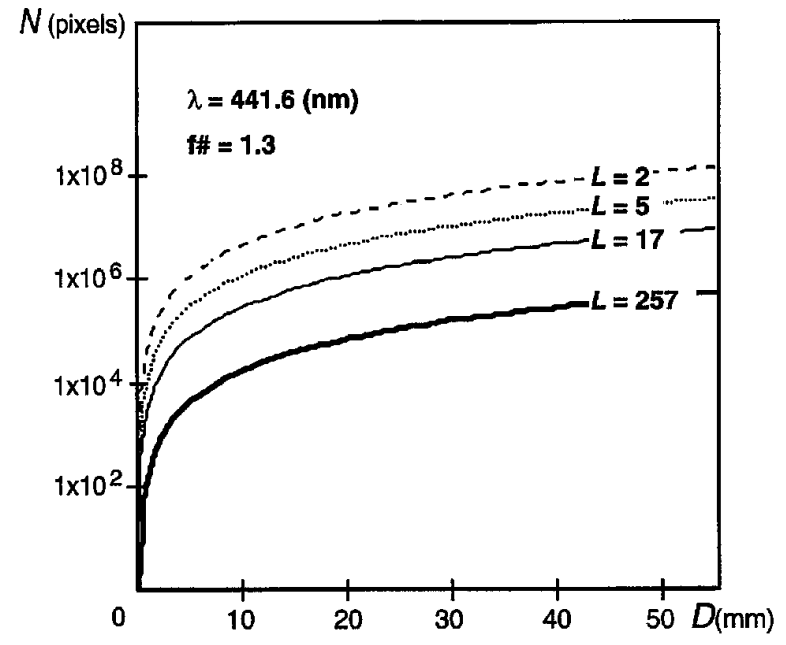

Fig. 12. Achievable pixel number $N$ as a function of the diameter of the lens, $D$, for $\lambda=441.6 \mathrm{~nm}$ and $f$-number $=1.30$. Cases $L=$ $2, L=5, L=17$, and $L=257$ are plotted.

real-time diagnosis of a system operation and a test of algorithms under development.

One practical issue of the gray-image morphology by the VACT is that the VACT wastes a lot of pixels in the processing. Thus we estimate the capacity of the optical system as shown in Fig. 9 in terms of the SBWP. In the system, the maximum image size $A_{\max }$ is given by

$$
A_{\max }=D-2 f \lambda v_{\max }
$$

where $D$ and $f$, are the diameter and the focal length of the lens $L$, respectively, $\lambda$ is the wavelength, and $v_{\max }$ is the maximum spatial frequency passing through the lens $L$. For simplicity, we consider the one-dimensional case. Then the SBWP is given by

$$
\mathrm{SBWP}=A_{\max } v_{\max }=-2 f \lambda\left(\nu_{\max }-\frac{D}{4 f \lambda}\right)^{2}+\frac{D^{2}}{8 f \lambda} .
$$

According to Eq. (2), the maximum SBWP, SBWP $_{\max }$, is given by $D^{2} /(8 f \lambda)$. Therefore an achievable pixel number $N$ for different gray levels is computed by

$$
N=\frac{\left(\mathrm{SBWP}_{\max }\right)^{2}}{L-1}=\frac{D^{4}}{64(L-1) f^{2} \lambda^{2}},
$$

where $L$ is the number of levels. Figure 12 plots the achievable pixel number for $\lambda=441.6 \mathrm{~nm}$ and $f$-number $=1.30$. For example, a gray image that consists of $500 \times 500$ pixels with 257 levels can be processed with $f=80.0 \mathrm{~mm}$ and $D=47.54 \mathrm{~mm}$.

\section{Conclusion}

We have presented a novel scheme of visible grayimage morphology with the VACT. The VACT is a technique of digitized analog-optical computing in which data are converted into visible coded patterns and processed with the visible form. Because the achievable operations in the VACT are identical to those of mathematical morphology, mathematical morphology has been adapted for gray-image morphology with the VACT. Computer simulation and optical experiments of several operations in mathematical morphology verify the correctness of the proposed technique. The processing capacity of the proposed method has been estimated in terms of the SBWP. The proposed method is expected to be applied to a human-aid system, a real-time diagnosis mechanism, and effective program test techniques.

\section{References}

1. J. Tanida and Y. Ichioka, "Optical logic array processor using shadowgrams," J. Opt. Soc. Am. 73, 800-809 (1983).

2. K.-H. Brenner, A. Huang, and N. Streibl, "Digital optical computing with symbolic substitution," Appl. Opt. 25, 30543060 (1986).

3. K.-S. Huang, B. K. Jenkins, and A. A. Sawchuk, "Image algebra representation of parallel optical binary arithmetic," Appl. Opt. 28, 1263-1278 (1989).

4. M. Fukui and K. Kitayama, "Applications of image-logic algebra: wire routing and numerical data processings,"Appl. Opt. 31, 581-591 (1992).

5. T. Konishi, J. Tanida, and Y. Ichioka, "Visual-area coding technique (VACT): optical parallel implementation of fuzzy logic and its visualization with the digital halftoning process," Appl. Opt. 34, 3097-3102 (1995).

6. P. Maragos, "Tutorial on advantages in morphological image processing and analysis," Opt. Eng. 26, 623-632 (1987).

7. E. Ochoa, J. P. Allebach, and D. W. Sweeney, "Optical median filtering using threshold decomposition," Appl. Opt. 26, 253260 (1987).

8. J. M. Hereford and W. T. Rhodes, "Nonlinear optical image filtering by time sequential threshold decomposition," Opt. Eng. 27, 274-279 (1988).

9. Y. Li, A. Kostrzewski, D. H. Kim, and G. Eichmann, "Compact parallel real-time programmable optical morphological image processor," Opt. Lett. 14, 981-983 (1989).

10. A. K. Cherri, A. A. S. Awwal, and M. A. Karim, "Morphological transformation using optical symbolic substitution," Microwave Opt. Technol. Lett. 2, 282-285 (1989).

11. P. Cambon and J.-L. Bougrenet de la Tocknaye, "Mathematical morphology processor using feroelectric liquid crystal light valves: principle,"Appl. Opt. 28, 3456-3460 (1989).

12. B. D. Duncan, T.-C. Poon, and R. J. Pieper, "Real-time nonlinear image processing using an active optical scanning technique," Opt. Laser Technol. 23, 19-24 (1991).

13. Z. Zhu and L. Liu, "Optical cellular continuous logic array for gray-scale image processing," Appl. Opt. 32, 3676-3683 (1993).

14. J. Gaecia, T. Szoplik, and X. H. Wang, "Optoelectronic morphological image processor,” Opt. Lett. 18, 1952-1954 (1993).

15. T. Szoplik, J. Garcia, and C. Ferreira, "Rank-order and morphological enhancement of image details with an optoelectronic processor," Appl. Opt. 34, 267-275 (1995).

16. L. Liu, "Optical implementation of parallel fuzzy logic," Opt. Commun. 73, 183-187 (1989).

17. F. Ono, "Binary rendition of continuous-tone pictures using binary patterns having similar Fourier spectra," Trans. Inst. Electron. Commun. Eng. Jpn. Part D J68D, 686-693 (1985).

18. P. Maragos and R. W. Schafer, "Morphological filters-Part II: their relations to median, order-statistic, and stack filters," IEEE Trans. Acoust. Speech Signal Process. ASSP-35, 11701184 (1987).

19. H. Tanigawa, T. Ichibashi, and A. Nagata, "Hologram recording on multicomponent monomer materials," Jpn. J. Opt. 20, 227-231 (1991). 